\title{
A SERVICE-IN-THE-LOOP APPROACH FOR BUSINESS PROCESS SIMULATION BASED ON MICROSERVICES
}

\author{
Paolo Bocciarelli \\ Andrea D'Ambrogio \\ Emiliano Paglia \\ Dept. Enterprise Engineering \\ University of Rome Tor Vergata \\ Viale del Politecnico 1 \\ 00133, Rome, Italy
}

\author{
Andrea Giglio \\ Dept. Innovation and Information Engineering \\ Guglielmo Marconi University \\ Via Plinio 44 \\ 00193, Rome, Italy
}

\begin{abstract}
An effective analysis of business processes (BPS) is crucial for modern organizations, which are required to promptly react to rapid market changes and evolving customer expectations. A suitable integration of standard BP management approaches and emerging technologies and methodologies, such as modeling and simulation $(M \& S)$, cloud computing and microservices, brings important strategic advantages in terms of cost reduction and efficiency, boosting the effectiveness of BP analysis activities. This paper discusses the $M S a a S$ ( $M \& S$ as a Service) paradigm, which aims at introducing in the M\&S domain innovative serviceoriented approaches based on microservices. The paper proposes an architecture of a MSaaS platform that enacts a service-in-the-loop approach, which properly combines real services and simulated ones for what-if analysis scenarios.
\end{abstract}

Keywords: MSaaS, BPMN, microservices, hybrid simulation, model-driven

\section{INTRODUCTION}

Modern organizations are required to implement an effective management of their business processes (BPs) if they want to be competitive on the global marketplace. Traditionally, business process management (BPM) approaches focus on standardization and continuous improvement, which are essential for those organizations that aim to increase the value of delivered products and services (Harrington 1991).

From a management perspective, BPM programs that require discovery, modeling, and analysis activities can result in long lead times, and businesses can no longer tolerate time-consuming implementations as they need to react quickly to dynamic market demand and changing customer expectations.

Also from an operational perspective, a proper integration of emerging technologies and BPM is needed to effectively support frequent deployments, multiple concurrent versions, and flexible scaling, also enabling IT assets to respond more quickly to the needs of the business.

The microservices software architecture paradigm is gaining momentum as an approach to enable a flexible execution and an independent deployment of service-oriented software systems (Karmel et al. 2016). A microservices architecture is characterized by a set of small services, running in their own processes and 
interacting through lightweight mechanisms, that together form a single application (Fowler, Martin and Lewis, James 2014). In the context of BPM, microservices can be usefully introduced to automate the execution of highly distributed business process models.

Modeling and Simulation $(M \& S)$ approaches have proven to be an effective solution to carry out the analysis of a BP behavior at both design and operation time, in order to evaluate the BP performance and compare design alternatives (D’Ambrogio et al. 2016, Bocciarelli et al. 2014). A typical BP exploits the interaction among activities executed by different organizations, thus implying a compositional and collaborative nature that easily leads to consider distributed simulation as a viable approach to properly address the analysis of BP collaborations, with collaborative BPs simulated as communicating process simulation components.

The effectiveness of M\&S approaches is widely recognized, yet there are still issues that limit their adoption in the BPM domain. Specifically, the non negligible effort and technical skills needed to build and implement BP simulation models (Barber et al. 2003, Melão and Pidd 2003), as well as the significant cost for establishing and maintaining a proper infrastructure for running distributed simulations.

The first issue (i.e., the cost of simulation model building and implementation) has been investigated by several authors, which eventually propose the adoption of model-driven engineering techniques as an effective solution to reduce the gap between BP conceptual modeling and simulation modeling (Cetinkaya et al. 2010, Kloos et al. 2009, Rust et al. 2011). Model-driven engineering is a design and implementation approach which emphasizes the formal definition of models and their automated transformation (Atkinson and Kühne 2003, Schmidt 2006). The Model Driven Architecture (MDA) is the OMG (Object Management Group) effort inspired by model-driven engineering principles (OMG 2003). A complete MDA-based framework has been proposed that automates both the generation of BP simulation models from BP models specified in BPMN and the BP simulation model implementation specified in the eBPMN domain-specific BP simulation language (Bocciarelli and D'Ambrogio 2012).

Cloud computing and service-oriented architectures help to properly address the second issue (i.e., the cost of simulation execution infrastructure). MSaaS ( $M \& S$ as a Service) is currently gaining momentum as an effective approach to bring the benefit of cloud computing and service-oriented architectures in the M\&S domain (Cayirci 2013).

The joint adoption of model-driven engineering approaches and MSaaS can thus be seen as a promising solution in order to: (i) reduce the effort of simulation model building, implementation, deployment and execution; and (ii) reduce the costs required to build and manage a distributed simulation execution infrastructure.

In previous contributions we discussed the state-of-the-art of M\&S approaches for BP analysis (Bocciarelli et al. 2017) and defined the requirements of a next generation MSaaS platform that exploits model-driven engineering approaches (Bocciarelli et al. 2017).

The purpose of this paper is to investigate the simulation-based analysis of BP collaborations and propose an extension of the aforementioned MSaaS platform based on the use of microservices. The proposed extension introduces a service-in-the-loop approach, in which the BP simulation may also include the use of real services. Such an approach may provide particular benefits at acquisition or procurement stage, when it is necessary to assess the impact on the BP collaboration of services to be selected according to the offered or required quality of service.

The rest of this paper is organized as follows: Section 2 provides the necessary background, while Section 3 introduces the MSaaS platform for simulation-based BP analysis and illustrates the service binding mechanism. Section 4 overviews the implementation of the service binding mechanism and, finally, Section 5 gives concluding remarks. 
Bocciarelli, D’Ambrogio, Giglio, and Paglia

\section{BACKGROUND}

This section provides the necessary background about the main elements addressed by this paper contribution, namely, BPMN, the underlying model-driven framework, microservices and containerization.

\subsection{BPMN}

The Business Process Model and Notation (BPMN) is an OMG specification that provides a standard graphical notation for the definition of BP models (OMG 2011). The primary goal of BPMN is to provide a notation that is easily understandable by all the various professional figures involved in the BP management, bridging the gap between the process design and the process implementation. Even though BPMN allows one to represent a BP at different levels of abstraction, it is mainly used in the analysis and design stages of the process lifecycle, but can be easily mapped to BP execution languages such as BPEL (Business Process Execution Language) (Ouyang et al. 2009, van der Aalst 2013).

BPMN provides three basic types of diagram for the graphical description of business processes: orchestration, choreography and collaboration, which in turn can include orchestrations and/or choreographies. The elements that a business process diagram can show are divided in four categories, flow objects, artifacts, connecting objects and swimlanes. Flow objects are the core elements provided by BPMN and include:

- events, which represent everything that could happen during the process and that have some relevance for the process flow;

- activities, which represent units of work performed at process execution time and can be either atomic (tasks) or compound (sub-processes);

- gateways, which are used to alter the sequential flow of control between objects. A gateway can be diverging or converging, thus specifying if it splits or merges the execution flow.

\section{2 eBPMN-based Model-Driven Framework}

The Model-Driven Architecture (MDA) (OMG 2003) is an effort promoted by the OMG to realize modeldriven engineering principles in the software development domain. MDA is based on a set of specifications to define metamodels (i.e., models that define modeling languages), to transform models and to serialize models into XML-based files (Mellor et al. 2004).

In previous contributions we have introduced a model-driven framework for the automated analysis of BPs specified in BPMN (Bocciarelli and D'Ambrogio 2012). The framework applies a set of model transformations that takes as input the BPMN model and eventually yields as output the simulation model implementation specified by use of the eBPMN language.

The eBPMN (executable BPMN) language (Bocciarelli et al. 2014) is a domain-specific simulation language that conforms to the execution semantics defined by the BPMN 2.0 specification, thus obtaining a compact and precise executable model of the business process model, without the need of introducing additional simulation formalisms, such as queueing-based modeling languages. As a consequence, it can be effectively used by business analysts who do not have specific simulation or programming know-how. Indeed, eBPMN has been designed with the aim of making BPMN models executable. The eBPMN language allows to simulate either a single process owned by a unique participant or a collaboration of processes that interact by use of message flow elements. In addition, eBPMN allows one to simulate the resource behavior and analyze the non-functional properties defined by the PyBPMN extension metamodel (Bocciarelli et al. 2016, Bocciarelli and D'Ambrogio 2011). 


\section{Bocciarelli, D’Ambrogio, Giglio, and Paglia}

The eBPMN language has been built on top of SimArch (Gianni et al. 2011), a layered software architecture that provides an event-driven simulation engine to execute simulation models in either local or distributed simulation environments. eBPMN consists of a set of Java-based primitives that implement the execution semantics of BPMN constructs.

The eBPMN language exploits the token concept to simulate the execution of a BPMN process. A token is produced at process instance starting, according to a given generation policy (typically an inter-arrival probability distribution). In this sense, a token can be considered as a reference to the execution of a process instance. Then, the token goes throughout the process elements, guided by sequence flows, and each eBPMN element handles the token according to its execution semantics.

eBPMN can be used to directly specify and execute BP simulation models, which can be also obtained in an automated fashion from standard BPMN models by use of the aforemention model-driven framework. In particular, a set of model-to-model and model-to-text transformations allow to generate the eBPMN simulation code including the non-functional properties that are specified as text annotations in the BPMN model. With respect to the specification of model transformations, eBPMN makes use of the Query/View/Transformation (QVT) standard (OMG 2015) for specifying model-to-model transformations and the MOF Model-to-Text (MOFM2T) standard (OMG 2008) for specifying model-to-text transformations.

The model-driven framework has been integrated into a cloud-based architecture that exploits the MSaaS paradigm and containerization technology for the flexible and dynamic composition of M\&S services, so to allow business analysts to carry out an effortless and effective M\&S-based BP analysis (Bocciarelli et al. 2017).

The rationale of the model-driven MSaaS platform is that a set of remote and cloud-based services can be selected and orchestrated in order to carry-out some of the tasks required to support the simulationbased analysis of a given system: a modeling service to specify the abstract model of the system; a set of transformation services to generate the corresponding executable simulation model; a simulation service to carry out the simulation-based analysis and, finally, a presentation service to summarize and show the related outcomes. The set of currently available transformation services can be found in (Bocciarelli et al. 2018).

\subsection{Microservices}

A microservice can be thought as a basic element obtained from the architectural decomposition of an application's components into loosely coupled patterns. Microservices consist of self-contained components that communicate with each other using a standard communication protocol and a set of well-defined Application Programming Interfaces (APIS) and events, independent of any vendor, product or technology (Karmel et al. 2016).

The microservices architecture is a pattern defined as "an approach to developing a single application as a suite of small services, each running in its own process and communicating with lightweight mechanisms" (Fowler, Martin and Lewis, James 2014). Microservices can be core or composite. In the latter case, a more complex service is realized as a composition of several core or composite services. These services communicate with each other through event-driven mechanisms primarily by asynchronous means through dumb message buses (i.e., communication logic is only present at the endpoints).

In a microservice architecture two fundamental aspects have to be addressed. On the one hand, distributed data management has to be managed in order to store a microservice state in local databases. On the other hand, shared event stores have to be supervised in order to facilitate the information exchange between state- 
less microservices. Information from local databases is thus used in conjunction with the event processing inside the microservice in order to execute the business logic.

The fundamental benefits of microservices can be summarized as follows:

- improved scalability: services can scale easily and independently from other services;

- improved agility: thanks to the high degree of modularity, it is possible to make a change to a service without impact on any other service;

- improved availability of the application: if a partial service failure occurs, it is highly unlikely that it will directly impact the rest of the application;

- continuous delivery: to make a change or update on a particular service, there is no need to set up the entire application in maintenance mode.

\subsection{Cloud Computing and Containerization}

Cloud computing is an information technology paradigm that enables ubiquitous access to shared pools of configurable system resources and higher-level services that can be provisioned in a rapid, dynamic, scalable and virtualized way with minimal management effort, often over the Internet. In a cloud-based infrastructure, the delivery paradigm allows computing resources to be provided on demand in a scalable and dynamic way. In more detail, cloud computing is founded on an everything-as-a-service delivery model that enables the provision of applications (Software-as-a-Service, SaaS), development platforms (platform-as-aService, PaaS) and computing infrastructures in terms of storing and processing capacity (Infrastructure-asa-Service, IaaS) (Menascé and Ngo 2009).

A basic building block of the cloud computing paradigm is the concept of containerization. A container is a self-contained runtime environment for a software application, which can be used to wrap services in order to ease their deployment and execution in the cloud. Containers provide a means to virtualize an operating systems so that multiple workloads can run on a single operating system instance, differently from virtual machines, which virtualize the hardware to run multiple operating system instances (Karmel et al. 2016).

Specifically, the proposed MSaaS platform adopts the Docker containerization technology (Docker 2016). A Docker container wraps a piece of software in a complete filesystem that contains everything needed to run the software: code, runtime, system tools, system libraries and anything that can be installed on a server, guaranteeing that the software will always run the same, regardless of its hosting environment (Docker 2016). It provides also native integration with many cloud service providers, such as Amazon AWS (Amazon 2017) or Microsoft Azure (Microsoft 2017).

The proposed MSaaS platform provides BP model execution in a microservices-based framework that makes use of containers and orchestrators. The container technology is used to execute the application code, whereas orchestrators automate the deployment of containerized applications.

\section{MICROSERVICES-BASED MSAAS ARCHITECTURE FOR BP ANALYSIS}

This section describes the proposed reference architecture for carrying out the analysis of BP collaborations by exploiting the potential of microservices.

As shown in Figure 1, the underlying concept that guides the proposed method is that a series of cloud-based services can be appropriately selected and orchestrated to perform the different activities that are necessary for the analysis of a BP. First, a modeling service is needed in order to specify the abstract model of the system; next, a set of transformation services enables the automated generation of the related executable 
simulation model (i.e., the simulator); then, a proper simulation service is used to actually execute the simulation; finally, a representation service allows to sum up and effectively present the simulation results to the user.

\subsection{Layered Architecture Overview}

The proposed platform has been designed according to a multi-tier architectural pattern. Specifically, the following different layers are identified, namely the Web Layer, through which the user is allowed to interact with the platform by means of a web client (i.e., a web browser), the Application Server Layer, which implements a series of components in order to provide access to the lower level that implements specific services and, finally, the Cloud Infrastructure Layer, responsible for the actual services implementation and containerization.

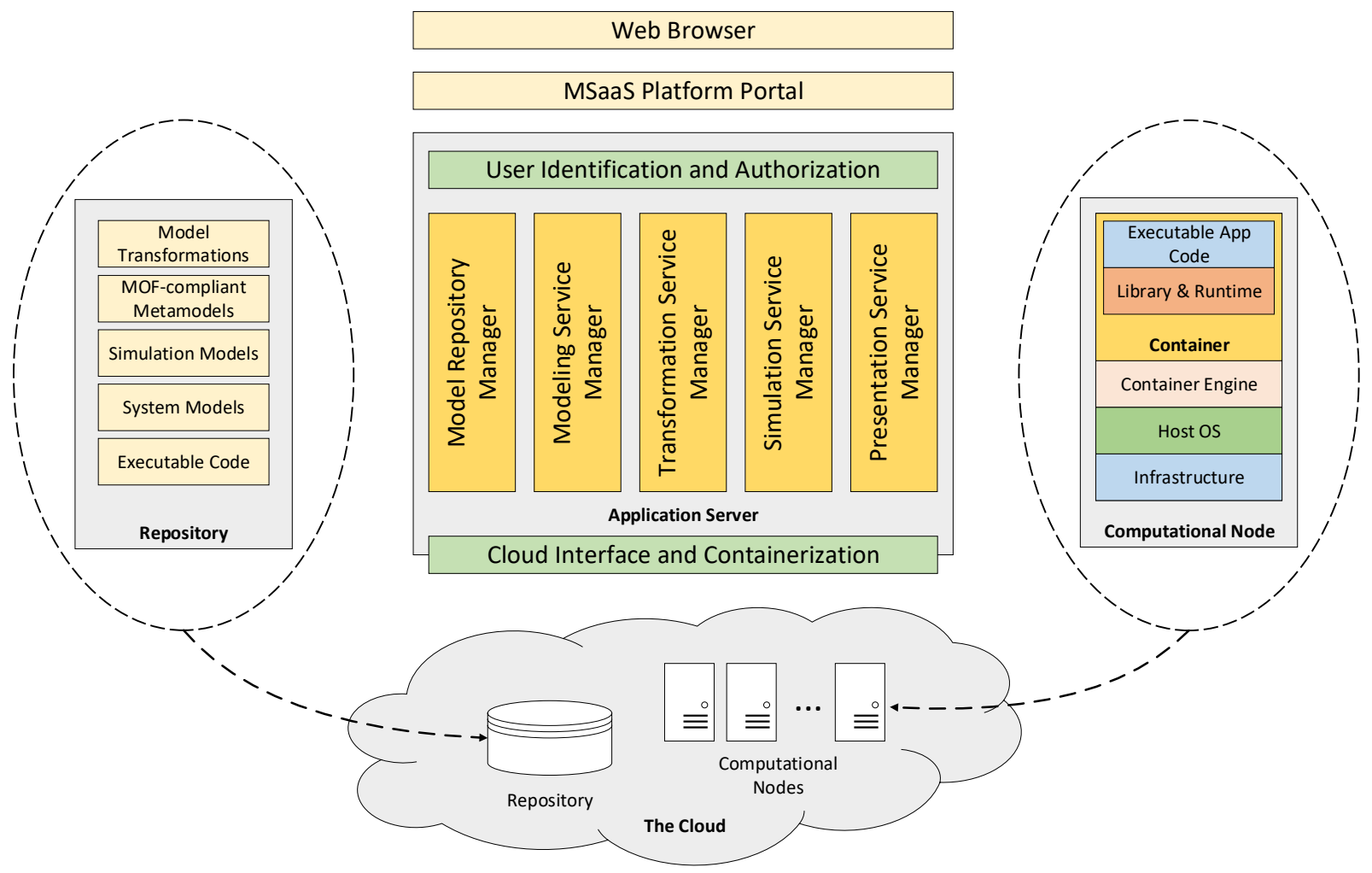

Figure 1: MSaaS Plaform Architecture.

The Application Server Layer provides the following set of components:

- User Identification and Authorization: this component is responsible for providing authentication services to the user.

- Model Repository Manager: this component is responsible for providing an interface to interact with the cloud-based repository (i.e., for storing and retrieving models).

- Modeling Service Manager: this component is responsible for providing modeling services to allow users to create, edit and store models. Modeling services can be seamlessly generated from different domain metamodels through the use of transformation services, thus empowering the flex- 
ibility of the proposed architecture, which is able to fit the needs of several application domains and modeling languages.

- Transformation Service Manager: this component is responsible for providing services that deal with model transformations storage, retrieval and execution. Such model transformations are used to generate both models and code, respectively. On the one hand, new models are obtained applying model-to-model transformations to existing models, on the other hand, executable modeling services or simulation services are obtained by use of model-to-text transformations from abstract models.

- Simulation Service Manager: this component is responsible for a set of key activities and is directly involved in the primary contribution of this paper. It is in charge of managing the required tasks needed to select a simulation service image available on the cloud repository, and deploy and execute it on the cloud infrastructure. The simulation Service Manager is also responsible for managing the simulation model partitioning, in case a distributed simulation execution platform is used. It is also responsible for the execution of the required model-to-text transformations to generate the code and the required configuration files, needed to execute the simulation model onto the simulation engine (i.e., binding a BP task to a specific service, as explained in the following subsection). Additionally, it is responsible for the actual deployment of the simulation components onto the target local or distributed simulation platform.

- Presentation Service Manager: this component is responsible for providing an effective visual representation of simulation results to the user.

- Cloud Interface: this component is responsible for providing the services for interacting with the underlying cloud infrastructure by use of a containerization approach.

The Cloud Infrastructure Layer provides the following set of components:

- Storage Service: this repository is responsible for the cloud-based storage of data related to M\&S services. Specifically, such data can be models, which can be either specified by use of a modeling service or generated by a model transformation, configurations data and services images. Being the executable code that implements a M\&S service, service images play a key role in the proposed platform. A given image can be the implementation of a simulation, a transformation or a modeling service and is stored in the aforementioned repository along with the required libraries and runtime, waiting to be deployed onto a proper container for execution.

- Computation Service: this component manages the computational nodes that define the actual infrastructure hosting the virtual containers (i.e. Docker containers) required to deploy and execute the aforementioned services.

\subsection{Service Binding}

This subsection gives a detailed discussion of the extended responsibilities that are provided by the Simulation Service Manager component of the proposed MSaaS platform. The concept of service binding is the key enabling factor for the service-in-the-loop approach, which enables the BP analyst to carry out a proper comparison between several services, considering their non-functional properties and evaluating their impact on the end-to-end BP performance, before proceeding with the services implementation or acquisition.

To this purpose, the primary contribution of this paper is to enrich the Simulation Service Manager with new capabilities that enact the service binding concept. Specifically, each task of a BP specified in a BPMN model is allocated to a given service implementation (i.e., the actual service to be invoked to execute the task). 


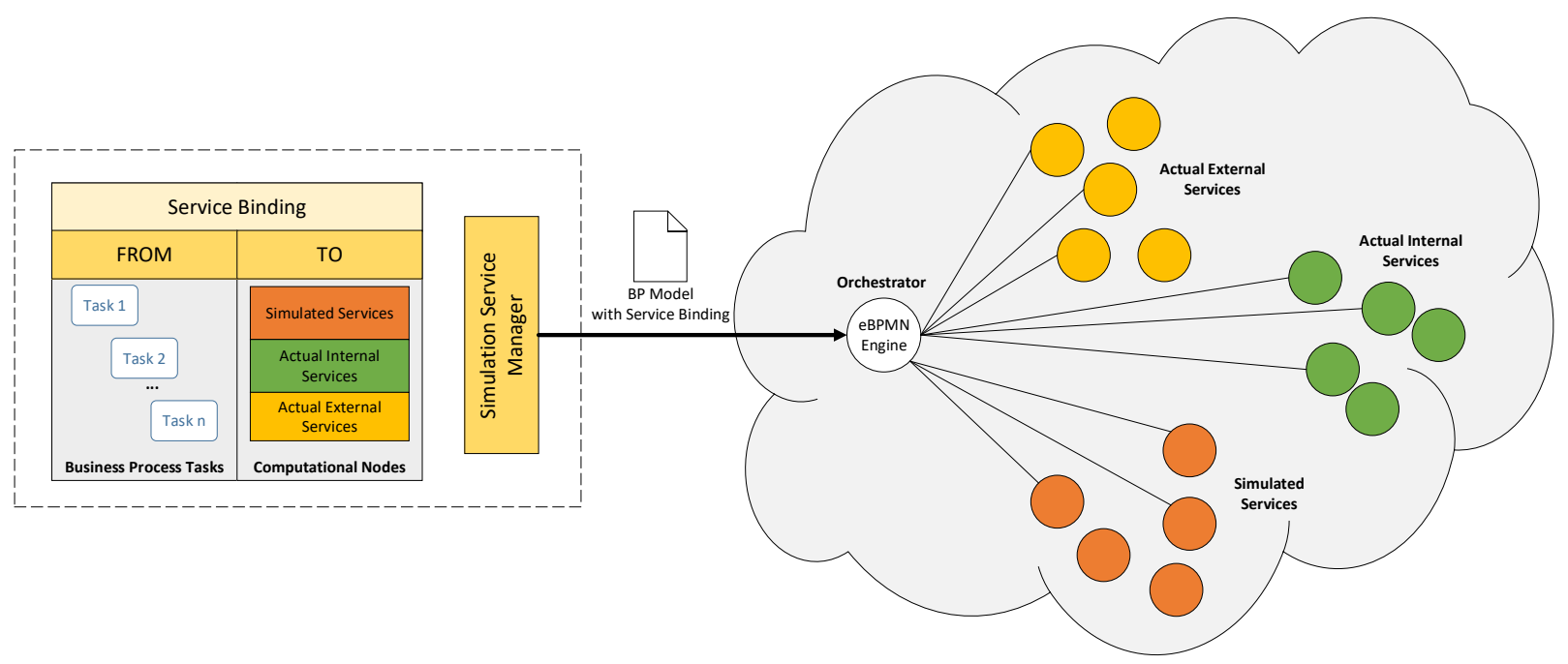

Figure 2: Simulation Service Manager and Service Binding.

Figure 2 depicts the concept of service binding as implemented by the Simulation Service Manager. It is assumed that the Simulation Service Manager receives as input a BPMN model from the Transformation Service Manager. Such a model is annotated with the service binding details for each BP task. Users specify the binding information in the form of standard BPMN text annotations.

The Simulation Service Manager identifies a granular association of each task to a specific service, and encapsulates this information in the simulation code and related configuration files, which are used to execute the simulation model. The so obtained BP simulation model, enriched with service binding information, is then sent to the eBPMN simulation engine.

In case of a BP analysis carried out on models specified in BPMN, the aforementioned engine is an eBPMN simulation service provided by the MSaaS platform. Since the engine is responsible to dispatch the simulation token towards the destination task, whenever an actual service has to be invoked, the engine can transform such token into a real service request. The response provided by the invoked service is then reverted into an eBPMN token, to be dispatched to the next simulated task.

In more detail, the service binding enacted by the simulation service manager is able to associate three different categories of services to a task, as depicted in Figure 2:

- Actual Internal Services: in this case the BP task is bound to an actual service, which is part of the same cloud infrastructure of the MSaaS platform.

- Actual External Services: in this case the BP task is bound to an actual service, which is not part of the same cloud infrastructure of the MSaaS platform. A third party service can be invoked by the simulation engine by means of standard calling mechanisms (e.g. REST APIs).

- Simulated Services: in this case the BP task is not bound to any actual service, since it is simulated by the eBPMN simulation engine.

It becomes clear that in such configuration, the eBPMN simulation engine is acting as a microservices orchestrator, since it is responsible for invoking different services that execute the corresponding BP tasks. The heterogeneity of the invoked services (i.e., actual or simulated) defines the nature of the proposed simulation-based BP analysis approach, which is referred to as a service-in-the-loop approach. 


\section{USAGE EXAMPLE}

The case study under consideration is a typical order management process. The process starts when a given user submits an order through a web site front-end. The process requires, as a first step, the checkout of the order. Then, the credit card information communicated by the user is verified, through a service provided by the bank, to ensure that the transaction is permitted, otherwise (e.g., the card number is wrong or the funds are not enough) the process ends. If the credit card verification is passed, the order is sent to the warehouse, where the invoice and the shipping requests are generated. Finally, an order confirmation is sent to the customer. The BPMN diagram of the process is depicted in Figure 3.

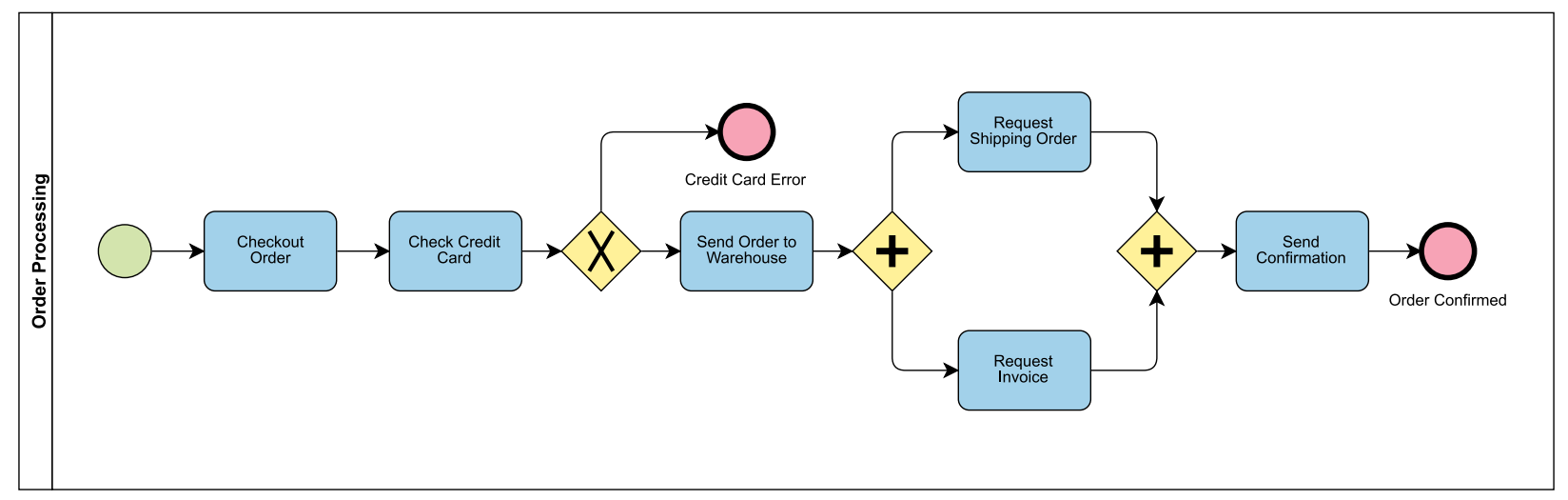

Figure 3: BPMN model for the order management process.

As an example, let us consider the Request Invoice task of the BP under study. This task has been bound to an external actual service by the simulation service manager, implying that a remote service has to be invoked by the simulation engine (i.e., the eBPMN engine in the proposed MSaaS platform). It is assumed that the customer id and the list of ordered items are available. Let us assume the following endpoint for a REST API of the example RequestInvoice microservice:

https://www.acmeshop.com/api/v64/8bitdivision/RequestInvoice/RequestInvoices

This API accepts a customer id and a list of items as an HTTP request payload. In this example we assume an XML encoding of such payload (JSON can be used as well):

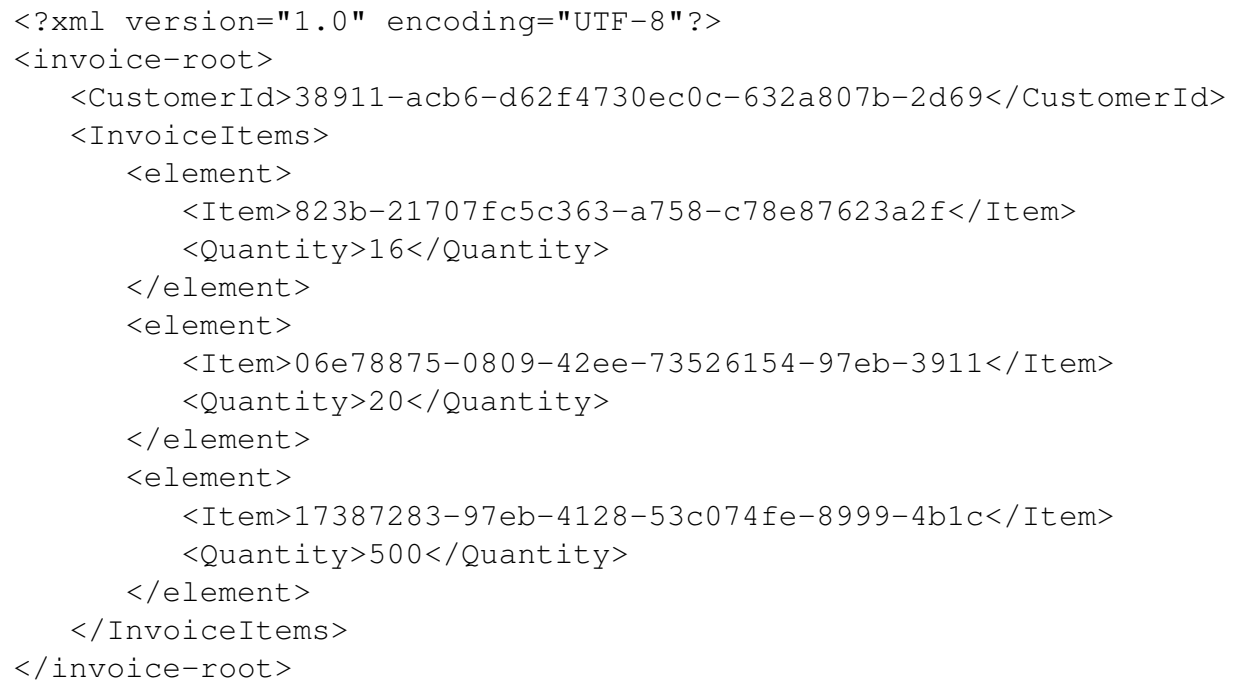


The relevant portion of a simple Java implementation of a microservice POST request via REST API, to obtain the invoice, is represented by the following listing:

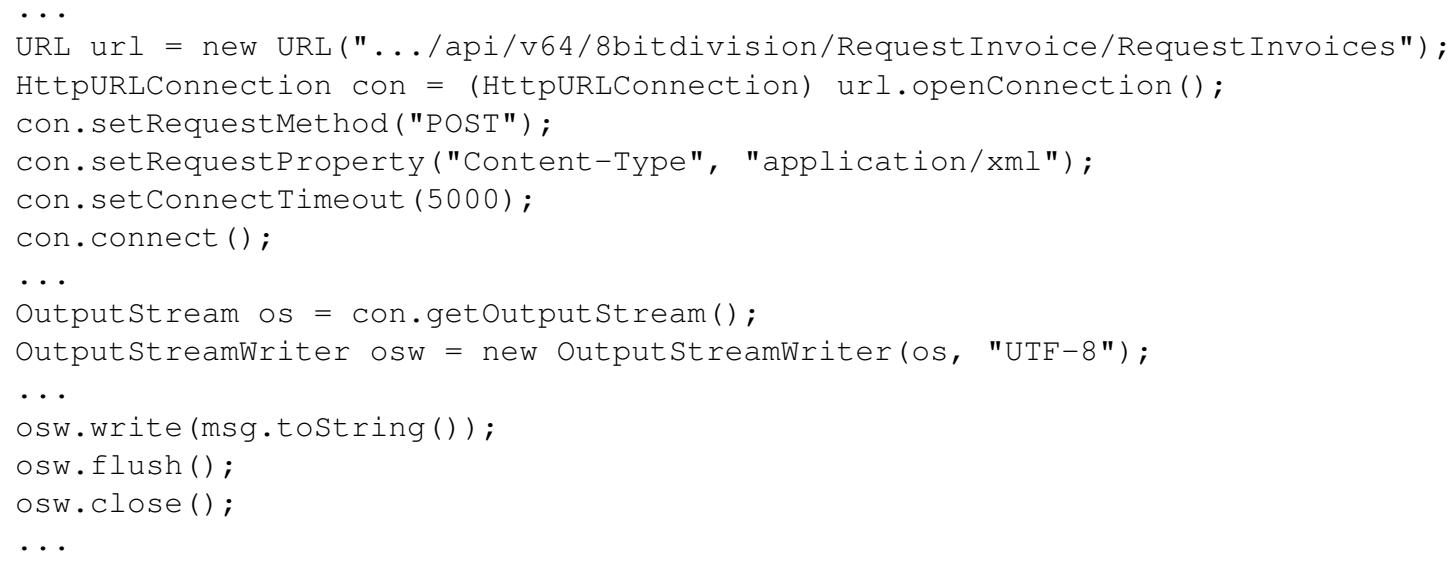

An HTTP connection is established specifying the API URL and setting the request method as POST and the content type as XML. Then, an output stream is used to send the payload (i.e., the XML document represented by the msg object).

An HTTP "201 Created" response is finally received, containing the resource identifier of the invoice.

\section{CONCLUSIONS}

The paper is focused on the MSaaS ( $M \& S$ as a Service) paradigm, which exploits the benefits of serviceoriented architectures and cloud-computing in the M\&S domain.

Specifically, the paper has introduced a MSaaS platform for the simulation-based analysis of BPs. The platform is founded on MDA-based model-driven technologies, to overcome the main issues which often limit an effective use of M\&S approaches in the BPM domain, and provides the capability of deploying microservices that implement the BP tasks. Moreover, in order to carry out simulation-based what-if analysis, the proposed platform enables BP analysts to make use of a service-in-the-loop approach, which properly combines simulated services and real services executing BP tasks.

Ongoing work includes an extensive testing phase of a full-featured implementation of the aforementioned MSaaS platform, in order to assess benefits and opportunities for improvement of the proposed approach.

\section{REFERENCES}

Amazon 2017. Amazon Web Services (AWS).

Atkinson, C., and T. Kühne. 2003. "Model-driven development: a metamodeling foundation". Software, IEEE vol. 20 (5), pp. 36-41.

Barber, K. D., F. W. Dewhurst, R. L. D. H. Burns, and J. B. B. Rogers. 2003. "Business-process modelling and simulation for manufacturing management: A practical way forward". Business Process Management Journal vol. 9 (4), pp. 527-542.

Bocciarelli, P., and A. D'Ambrogio. 2011. "A model-driven method for describing and predicting the reliability of composite services”. Software and Systems Modeling vol. 10 (2), pp. 265-280.

Bocciarelli, P., and A. D’Ambrogio. 2012. "Automated Performance Analysis of Business Processes". In Proceedings of the 2012 Symposium on Theory of Modeling and Simulation - DEVS Integrative M\&S 
Symposium, TMS/DEVS '12, pp. 10:1-10:9. San Diego, CA, USA, Society for Computer Simulation International.

Bocciarelli, P., A. D' Ambrogio, A. Giglio, and E. Paglia. 2014. "Simulation-Based Performance and Reliability Analysis of Business Processes". In Proceedings of the 2014 Winter Simulation Conference, pp. 3012-3023. Savannah, Georgia, IEEE Press.

Bocciarelli, P., A. D' Ambrogio, A. Giglio, and E. Paglia. 2016. "A BPMN extension to enable the explicit modeling of task resources". Volume 1728, pp. 40-47, CEUR-WS. Proceedings of the2nd INCOSE Italia Conference on Systems Engineering (CIISE 2016), Turin, Italy, November 14-16, 2016.

Bocciarelli, P., A. D’ Ambrogio, A. Giglio, and E. Paglia. 2018. "Model Transformation Services for MSaaS Platforms". In Proceedings of the Model-driven Approaches for Simulation Engineering Symposium, Mod4Sim '18, pp. 12:1-12:12. San Diego, CA, USA, Society for Computer Simulation International.

Bocciarelli, P., A. D’ Ambrogio, A. Giglio, E. Paglia, and T. Panetti. 2017. “A Cloud-based Service-oriented Architecture for Business Process Modeling and Simulation". In Proceedings of the 3rd INCOSE Italia Conference on Systems Engineering, Naples, Italy, November 22-24, 2017, pp. 87-93. Naples, Italy.

Bocciarelli, P., A. D'Ambrogio, A. Mastromattei, and A. Giglio. 2017. "Automated Development of Webbased Modeling Services for MSaaS Platforms". In Proceedings of the Symposium on Model-driven Approaches for Simulation Engineering, Mod4Sim '17, pp. 8:1-8:12. San Diego, CA, USA, Society for Computer Simulation International.

Bocciarelli, P., A. D’Ambrogio, A. Mastromattei, E. Paglia, and A. Giglio. 2017. "Business Process Modeling and Simulation: State of the Art and MSaaS Opportunities". In Proceedings of the Summer Simulation Multi-Conference, SummerSim '17, pp. 6:1-6:12. San Diego, CA, USA, Society for Computer Simulation International.

Bocciarelli, P., A. D’Ambrogio, and E. Paglia. 2014. "A Language for Enabling Model-Driven Analysis of Business Processes". In Proceedings of the 2nd International Conference on Model-Driven Engineering and Software Development, MODELSWARD'14. Lisbon, Portugal, SciTePress.

Cayirci, E. 2013. "Modeling and simulation as a cloud service: a survey". In Proceedings of the 2013 Winter Simulation Conference, WSC '13, pp. 389-400. San Diego, CA, USA, Society for Computer Simulation International.

Cetinkaya, D., A. Verbraeck, and M. Seck. 2010. “Applying a Model Driven Approach To Component Based Modeling and Simulation". In Proceedings of the 2010 Winter Simulation Conference, pp. 546-553.

D’Ambrogio, A., E. Paglia, P. Bocciarelli, and A. Giglio. 2016, April. "Towards performance-oriented perfective evolution of BPMN models". In 2016 Symposium on Theory of Modeling and Simulation (TMSDEVS), pp. 1-8.

Docker 2016. "Docker - Build, Ship, and Run Any App, Anywhere". Website: https://www.docker.com.

Fowler, Martin and Lewis, James 2014. "Microservices". Website: http://martinfowler.com/articles/ microservices.html.

Gianni, D., A. D’Ambrogio, and G. Iazeolla. 2011. "A software architecture to ease the development of distributed simulation systems". Simulation vol. 87 (9), pp. 819-836.

Harrington, H. 1991. "Business process improvement: The breakthrough strategy for total quality, productivity, and competitiveness". Journal of public health management and practice vol. 16, pp. 104-9.

Karmel, A., R. Chandramouli, and M. Iorga. 2016. NIST Special Publication 800-180: NIST Definition of Microservices, Application Containers and Virtual Machines. 
Kloos, O., V. Nissen, and M. Petsch. 2009. "From Process to Simulation - A Transformation Model Approach". In Proceedings of the 3rd International Workshop on Enterprise Modelling and Information Systems Architectures, pp. 83-96.

Melão, N., and M. Pidd. 2003. "Use of business process simulation: A survey of practitioners". Journal of the Operational Research Society vol. 54 (1), pp. 2-10.

Mellor, S. J., K. Scott, A. UHL, and D. Weise. 2004. MDA Distilled - Principles of Model-Driven Architecture. Addison Wesley.

Menascé, D. A., and P. Ngo. 2009. "Understanding Cloud Computing: Experimentation and Capacity Planning”. In Proceedings of the 2009 Computer Measurement Group Conference.

Microsoft 2017. Microsoft Azure.

OMG 2003. MDA Guide, version 1.0.1.

OMG 2008. MOF Model to Text Transformation Language.

OMG 2011. Business Process Modeling Notation (BPMN), version 2.0.

OMG 2015. "Meta Object Facility (MOF) 2.0 Query/View/Transformation".

Ouyang, C., M. Dumas, W. M. P. van der Aalst, and A. H. M. ter Hofstede. 2009. "From business process models to process-oriented software systems". ACM Transaction on Software Engineering Methodology vol. 19, pp. 1-37.

Rust, I., D. Cetinkaya, M. Seck, and I. Wenzler. 2011. "Business Process Simulation for Management Consultants: a Devs-Based Simplified Business Process Modelling Library". In Proceedings of the 23rd European Modelling and Simulation Symposium (EMSS 2011).

Schmidt, D. C. 2006. "Model-Driven Engineering". IEEE Computer vol. 39 (2).

van der Aalst, W. M. P. 2013. "Business Process Management: A Comprehensive Survey". ISRN Software Engineering vol. 2013, pp. 1-37.

\section{AUTHOR BIOGRAPHIES}

PAOLO BOCCIARELLI is a postdoc researcher at the University of Rome Tor Vergata (Italy). His research addresses the application of $M \& S$ and model-driven development to software and systems engineering and business process management. His email address is paolo.bocciarelli@uniroma2.it.

ANDREA D'AMBROGIO is associate professor at the Dept. of Enterprise Engineering of the University of Roma Tor Vergata (Italy). His research interests are in the fields of model-driven software engineering, dependability engineering, distributed and web-based simulation. His email address is dambro@uniroma2.it.

ANDREA GIGLIO is assistant professor at the "Guglielmo Marconi" University. His research interests include model-driven system and software engineering and business process management. His email address is a.giglio@unimarconi.it.

EMILIANO PAGLIA is a postdoc researcher at the University of Rome Tor Vergata (Italy). His research interests include model-driven engineering and business process modeling and analysis. His email address is emiliano.paglia@uniroma2.it. 